\title{
UNIQUENESS AND STABILITY OF SOLUTIONS FOR A TYPE OF PARABOLIC BOUNDARY VALUE PROBLEM
}

ENRIQUE A. GONZALEZ-VELASCO

Department of Mathematics

University of Lowe 11

Lowe 1, Massachusetts, U.S.A.

(Received June 25, 1986 and in revised form October 10, 1986)

\begin{abstract}
We consider a boundary value problem consisting of the one-dimensional parabolic equation $g u_{t}=\left(h u_{x}\right)_{x}+q$, where $g, h$ and $q$ are functions of $x$, subject to some general boundary conditions. By developing a maximum principle for the boundary value problem, rather than the equation, we prove the uniqueness of a nonnegative solution that depends continuously on boundary values.
\end{abstract}

KEY WORDS AND PHRASES. Parabolic equations, maximum principles. 1980 AMS SUBJECT CLASSIFICATION CODE. 35K20.

1. INTRODUCTION.

If a is a positive constant, $D=\left\{(x, t) \varepsilon \mathbb{R}^{2}: 0<x<a, t>0\right\}$ and
$\bar{D}=\left\{(x, t) \varepsilon \mathbb{R}^{2}: 0<x<a, t>0\right\}$, we shall be concerned with continuous nonnegative solutions $u: \bar{D} \rightarrow$ IR of the parabolic boundary value problem

$$
\begin{array}{ll}
g u_{t}=\left(h u_{x}\right)_{x}+q & \text { in } D \\
a_{1} u(0, t)-b_{1} u_{x}(0, t)=c_{1} & t>0 \\
a_{2} u(a, t)+b_{2} u_{x}(a, t)=c_{2} & t>0 \\
u(x, 0)=f(x) & 0<x<a
\end{array}
$$

where we assume that

(i) $g, h$ and $q$ are nonnegative continuous functions on $[0, a], g$ and $h$ never vanish and $h$ has a continuous derivative,

(ii) $a_{1}, a_{2}, b_{1}, b_{2}, c_{1}$ and $c_{2}$ are nonnegative constants,

(iii) $a_{1}+b_{1}>0$ and $a_{2}+b_{2}>0$, so that none of the boundary conditions is missing,

(iv) $a_{1} a_{2}>0$, and

(v) $f$ is a nonnegative continuous function with a piecewise continuous derivative and satisfies (1.2) and (1.3). 
A continuous solution on $\bar{D}$ must be bounded below, and we are assuming without loss of generality that this bound is zero. This is in agreement with the constraints on the constants $c_{1}$ and $c_{2}$.

The question of existence of solutions is easily answered by finding first the steady state solution and then using separation of variables and the Sturm-Liouville theory to prove the existence of a transient solution. It can be shown that a nonnegative steady state solution always exists under the stated conditions while, if condition (iv) falls to hold, it may exist but with probability zero. For proof see [1]. When $a_{1} a_{2}>0$ it may still not exist if either $b_{1}<0$ or $b_{2}<0$, as in the problem

$$
\begin{aligned}
& u_{t}=u_{x x} \\
& u(0, t)+u_{x}(0, t)=1 \\
& 2 u(1, t)=1
\end{aligned}
$$

which admits no solution independent of time. In fact, under these conditions it cannot even be guaranteed that the solution will be nonnegative, as in the problem

$$
\begin{aligned}
& u_{t}=u_{x x} \\
& u(0, t)-u_{x}(0, t)=3 \\
& u(1, t)-u_{x}(1, t)=1
\end{aligned}
$$

whose only solution independent of time is $U(x)=1-2 x$.

Uniqueness of solutions is sometimes obtained from a strong maximum principle of A. Friedman [2], for a general type of parabolic equation. In this paper we shall be able to give a much shorter proof by developing, instead, a type of maximum principle for the boundary value problem, rather than for the equation alone. This will provide the added benefits of enabling us to prove that the unique solution is nonnegative and depends continuously on the boundary and initial values. In the simpler case in which (1.1) becomes the heat equation, all of these facts are easily concluded from the usual maximum principle of E.E. Levi, but such a theorem is not valid when $q \neq 0$. However, the following starting statement about the minimum of a solution will be sufficient for our purposes.

\section{MAIN RESULTS.}

THEOREM 1. Let $\mathrm{T}$ be an arbitrary positive number and define the sets

$$
\begin{aligned}
& \mathrm{D}_{\mathrm{T}}=\{(\mathrm{x}, \mathrm{t}) \varepsilon \mathrm{D}: \mathrm{t}<\mathrm{T}\} \\
& \overline{\mathrm{D}}_{\mathrm{T}}=\{(\mathrm{x}, \mathrm{t}) \varepsilon \overline{\mathrm{D}}: \mathrm{t}<\mathrm{T}\}
\end{aligned}
$$

and

$$
\gamma_{\mathrm{T}}=\left\{(\mathrm{x}, \mathrm{t}) \in \overline{\mathrm{D}}_{\mathrm{T}}:(\mathrm{x}, \mathrm{t}) \notin \mathrm{D}_{\mathrm{T}}\right\}
$$


If $u: \bar{D}_{T}+\mathbb{R}$ is a continuous function that satisfies (1.1) in $D_{T}$, then u attains its minimum value on $\gamma_{T}$.

The proof is a trivial modification of that of Levi's principle.

THEOREM 2. Let $D_{T}, \bar{D}_{T}, \gamma_{T}$ and $u$ be as in Theorem 1 and define the sets

$$
\gamma_{0}=\left\{(x, t) \& \gamma_{T}: x=0, t>0\right\}
$$

and

$$
\gamma_{a}=\left\{(x, t) \& \gamma_{T}: x=a, t>0\right\} .
$$

If $u$ has a negative minimum on $\bar{D}_{T}$ and if

1. u satisfies (1.2), then it attains its minimum on $\gamma_{T}-\gamma_{0}$.

2. u satisfies (1.3), then it attains minimum on $\gamma_{T}-\gamma_{a}$.

PROOF. By Theorem $1, u$ attains its minimum value $m<0$ on $\gamma_{T}$.

1. Assume that, contrary to the desired conclusion, $u\left(0, t_{0}\right)=m$ for some $t_{0}>0$ while $u>M>m$ on $\gamma_{T}-\gamma_{0}$. Then $u_{x}\left(0, t_{0}\right)>0$ and $a_{1} u\left(0, t_{0}\right)-b_{1} u_{x}\left(0, t_{0}\right)<0$, which is impossible if $c_{1}>0$. Thus, $c_{1}=0$ and then $b_{1}>0$, or else $a_{1} u\left(0, t_{0}\right)=a_{1} m<0$, contradicting the value of $c_{1}$.

If we now define

$$
H(x)=\int_{0}^{x} \frac{1}{h}
$$

and a new function $v: \bar{D}_{T} \rightarrow \mathbb{R}$ by

$$
v(x, t)=u(x, t)-\frac{M-m}{2 H(a)} H(x)
$$

then $v$ is continuous and satisifies (1.1) because

$$
\begin{aligned}
g v_{t}-\left(h v_{x}\right)_{x} & =g u_{t}-\left(h u_{x}\right)_{x}-\left(h \frac{M-m}{2 H(a)} \frac{1}{h}\right)_{x} \\
& =g u_{t}-\left(h u_{x}\right)_{x}=q
\end{aligned}
$$

According to Theorem $1, v$ also attains its minimum on $\gamma_{T^{*}}$ In fact, this minimum is at $\left(0, t_{0}\right)$ because if $(x, t) \varepsilon \gamma_{T}-\gamma_{0}$ then

$$
v(x, t)>u(x, t)-\frac{M-m}{2}>M-\frac{M-m}{2}=\frac{M+m}{2}>m
$$

while $\mathrm{v} \equiv \mathrm{u}$ on $\gamma_{0}$.

Now, since $c_{1}=0$ and $b_{1}>0$, differentiation of $v$ with respect to $x$ gives

$$
\begin{aligned}
& v_{x}\left(0, t_{0}\right)=u_{x}\left(0, t_{0}\right)-\frac{M-m}{2 H(a) h(0)} \\
& =\frac{a_{1}}{b_{1}} u\left(0, t_{0}\right)-\frac{M-m}{2 H(a) h(0)}<0
\end{aligned}
$$

Then $v$ is decreasing in the $x$ direction at $\left(0, t_{0}\right)$, contradicting the fact that it has its minimum at this point. This contradiction shows that $u$ must attain its minimum on $\gamma_{T}-\gamma_{0}$ 
2. The proof is analogous to the one above, but based on the choice

$$
v(x, t)=u(x, t)-\frac{M-m}{2 H(a)}[H(a)-H(x)]
$$

Q.E.D.

These theorems have two corollaries that are applicable to solutions of

$$
\begin{array}{ll}
g u_{t}=\left(h u_{x}\right)_{x} & \text { in } D \\
a_{1} u(0, t)-b_{2} u_{x}(0, t)=0 & t>0 \\
a_{2} u(a, t)+b_{2} u_{x}(a, t)=0 & t>0
\end{array}
$$

and we shall use them to complete the discussion of our boundary value problem.

COROLlaRY 1. A continuous function $u: \bar{D}_{T}+\mathbb{R}$ that satisfies $(2.1)$ in $D_{T}$ attains its maximum value on $\gamma_{\mathrm{T}}$. If this maximum value is positive and if

1. u satisfies (2.2), then it attains its maximum on $\gamma_{T}-\gamma_{0}$.

2. u satisfies (2.3), then it attains its maximum on $\gamma_{T}-\gamma_{a}$.

PROOF. If $u$ satisfies any of the last three equations, so does $-u$, and thus $-u$ satisfies the hypotheses of Theorems 1 and 2 with $q \equiv 0$, and $c_{1}=c_{2}=0$. Accordingly, the statements made by these theorems about the minimum of -u translate into the statements made here about the maximum of $u, Q . E . D$.

COROLLARY 2. Let $u: \bar{D} \rightarrow \mathbb{R}$ be a continuous function that satisifes (2.1) (2.3). If $M_{0}$ and $m_{0}$ denote its maximum and minimum values on $\gamma_{T}-\left\{\gamma_{0} \cup \gamma_{a}\right\}$, then

$$
\min \left\{0, \mathrm{~m}_{0}\right\}<\mathrm{u}(\mathrm{x}, \mathrm{t})<\max \left\{0, \mathrm{M}_{0}\right\}
$$

for all $(x, t) \varepsilon \bar{D}$.

PROOF. Let $T>0$ be arbitrary. By Theorem 1 , the restriction of $u$ to $\bar{D}_{T}$ attains its minimum value $m$ on $\gamma_{\mathrm{T}}$. Either $m>0$, and then $u(x, t)>0$, or $m<0$. In this second case, Theorem 2 implies that $u$ attains its minimum on $\gamma_{\mathrm{T}}-\left\{\gamma_{0} \cup \gamma_{\mathrm{a}}\right\}$ and thus $m=m_{0}$ and $u(x, t)>m_{0}$. Since $T$ is arbitrary, this proves the inequality on the left. Similarly, by Corollary 1 , the restriction of $u$ to $\bar{D}_{T}$ attains its maximum value $M$ on $Y_{T}$. Either $M<0$, and then $u(x, t)<0$, or $M>0$. In this second case, and again by Corollary 1 , $u$ attains its maximum on $\gamma_{T}-\left\{\gamma_{0} \cup \gamma_{a}\right\}$, and thus $M=M_{0}$ and $u(x, t)<M_{0} \cdot$ Since $T$ is arbitrary, this proves the inequality on the right, Q.E.D.

We now return to the original boundary value problem.

THEOREM 3. The boundary value problem (1.1) - (1.4) has a unique continuous solution $u: \bar{D}+\mathbb{R}$ that depends continuously on initial values, and this solution is nonnegtive.

PROOF. If $u_{1}$ and $u_{2}$ are solutions of (1.1) - (1.4) corresponding to initial functions $f$ and $g$, then $u_{1}-u_{2}$ satisfies (2.1) - (2.2) and the new initial condition

$$
\left(u_{1}-u_{2}\right)(x, 0)=f(x)-g(x) \text {. }
$$


If $M_{0}$ and $m_{0}$ denote the maximum and minimum values of $f-g$, then Corollary 2 implies that

$$
\min \left\{0, m_{0}\right\}<\left(u_{1}-u_{2}\right)(x, t)<\max \left\{0, M_{0}\right\}
$$

for all $(x, t) \varepsilon \bar{D}$. Therefore, if $f$ is close to $g$ on $[0, a], M_{0}$ and $m_{0}$ are close to zero and so is $u_{1}-u_{2}$. This proves the continuity of solutions with respect to initial conditions and, taking $f=g$, proves uniqueness.

Finally, if $u$ has negative values the $n$, according to Theorem 2 , it attains its negative minimum on $\gamma_{T}-\left\{\gamma_{0} \cup \gamma_{a}\right\}$, where it equals $f$. But this is impossible because f $>0$. Q.E.D.

To prove the continuity of the solution with respect to both boundary and initial conditions, notice that the steady state solution $U$ as found in [1],

where

$$
\begin{aligned}
U(x)= & -\int_{0}^{x} \frac{Q}{h}+\frac{h(0) H(x)}{\Delta}\left[\left(a_{1} c_{2}-a_{2} c_{1}+a_{1} a_{2} \int_{0}^{a} \frac{Q}{h}\right) h(a)+a_{1} b_{2} Q(a)\right] \\
& +\frac{l}{\Delta}\left(b_{1} c_{2} h(a)+b_{2} c_{1} h(0)+b_{1} b_{2} Q(a)+a_{2} c_{1} h(0) h(a) H(a)+a_{2} b_{1} h(a) \int_{0}^{a} \frac{Q}{h}\right)
\end{aligned}
$$

and

$$
Q(x)=\int_{0}^{x} q
$$

$$
\Delta=\left[a_{1} h(0) H(a)+b_{1}\right] a_{2} h(a)+a_{1} b_{2} h(0)
$$

depend continuously on the values of the constants. Then, if $u$ is the unique solution of $(1.1)-(1.4)$ and we define $v=u-U$, this function satisfies (2.1) - (2.3). As above, $v$ depends continuously on $f-U$, which, in turn, depends continuously on $f$ and the constants. That is $u$ is stable with respect to initial and boundary values. We can sum up our results as follows

THEOREM 4. The boundary value problem (1.1) - (1.4) is well posed in the sense of Had ama $\mathrm{d}$.

\section{REFERENCES}

1. GonZalez-VElasco, E.A., The Existence of a Steady State Solution for a Type of Parabolic Boundary Value Problem, Int. J. Math. Educ. in Sci. \& Technol. 19(3), (1988), 413-419.

2. PROTTER, M.H. and WEINBERGER, H.F., Maximum Principles in Differential Equations, Prentice Hal 1, 1967. 


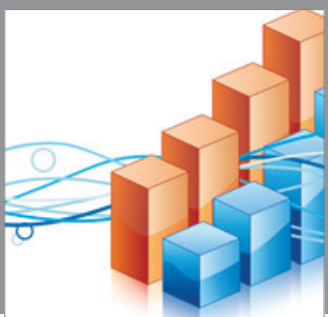

Advances in

Operations Research

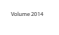

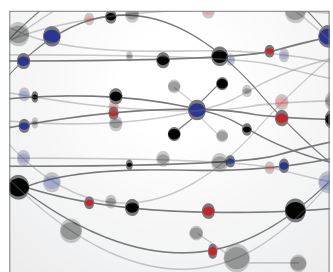

\section{The Scientific} World Journal
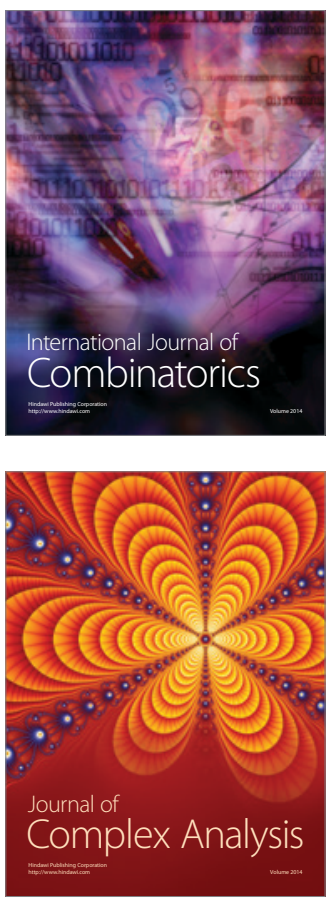

International Journal of

Mathematics and

Mathematical

Sciences
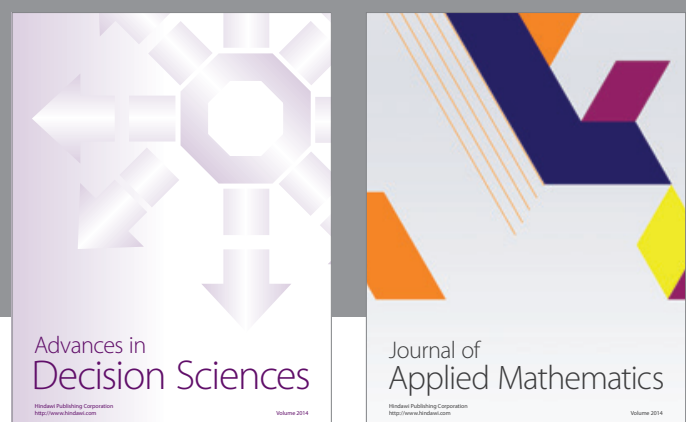

Journal of

Applied Mathematics
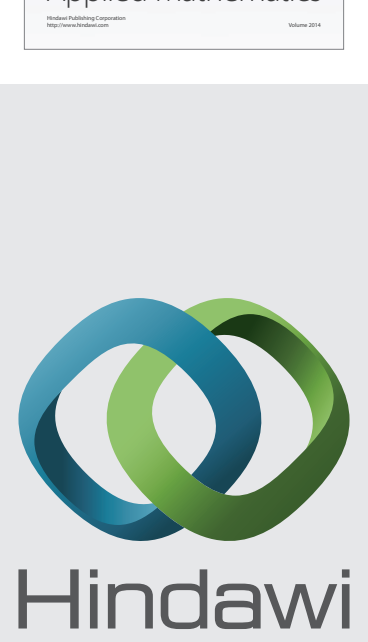

Submit your manuscripts at http://www.hindawi.com
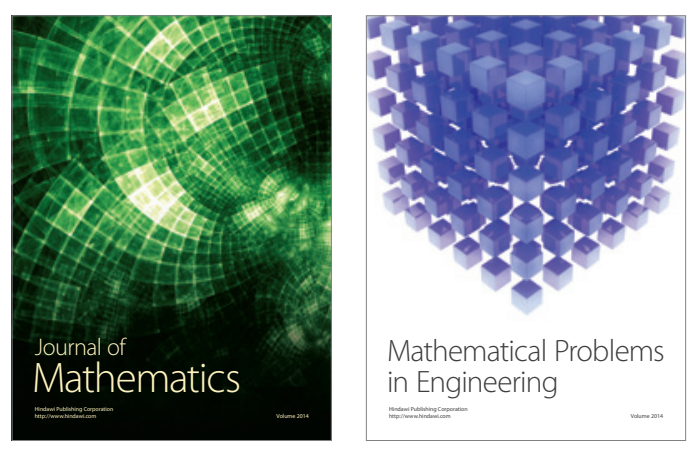

Mathematical Problems in Engineering
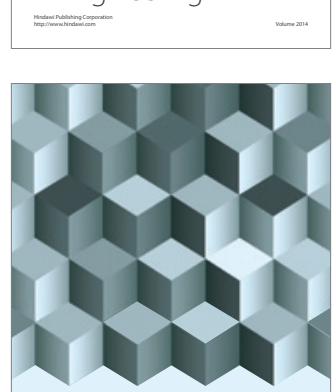

Journal of

Function Spaces
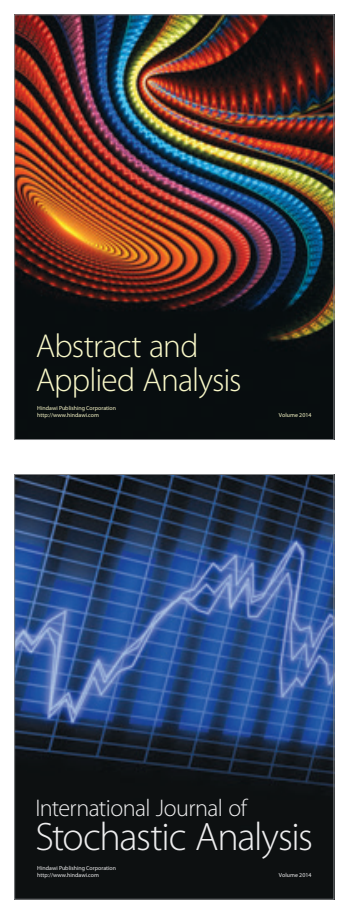

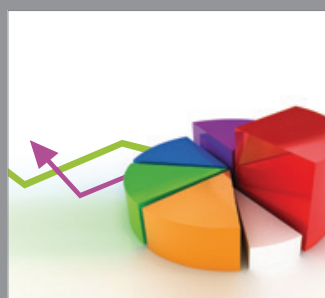

ournal of

Probability and Statistics

Promensencen
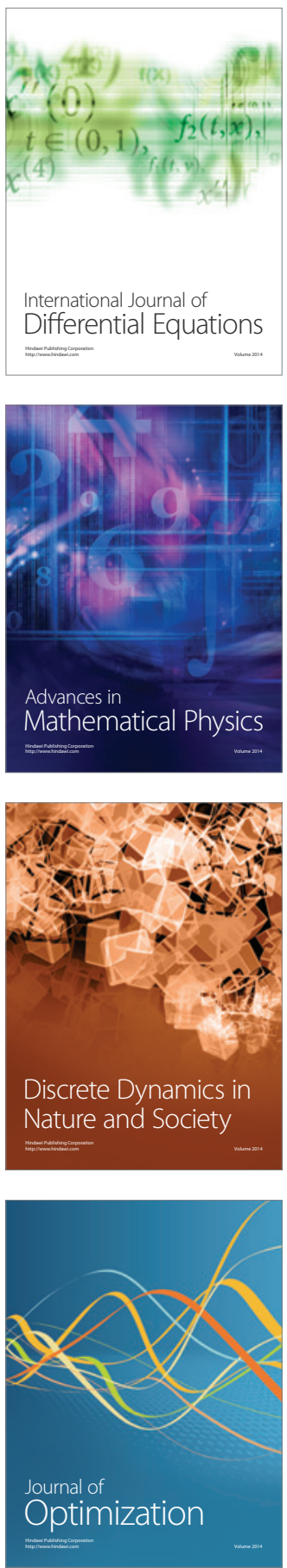